\title{
Subcutaneous Administration of D-Luciferin is an Effective Alternative to Intraperitoneal Injection in Bioluminescence Imaging of Xenograft Tumors in Nude Mice
}

\author{
Ashraf A. Khalil, ${ }^{1,2}$ Mark J. Jameson, ${ }^{1}$ William C. Broaddus, ${ }^{3}$ Theodore D. Chung, \\ Sarah E. Golding, ${ }^{5}$ Seth M. Dever, ${ }^{5}$ Elisabeth Rosenberg, ${ }^{5}$ and Kristoffer Valerie ${ }^{5}$ \\ ${ }^{1}$ Department of Otolaryngology-Head and Neck Surgery, University of Virginia Health System, P.O. Box 800713, \\ Charlottesville, VA 22908-0713, USA \\ ${ }^{2}$ Department of Biochemistry, National Liver Institute, Menoufiya University, Shebin Elkom 32511, Egypt \\ ${ }^{3}$ Department of Neurosurgery, Virginia Commonwealth University, Richmond, VA 23298, USA \\ ${ }^{4}$ Department of Radiation Oncology, Georgia Health University, Augusta, GA 30912, USA \\ ${ }^{5}$ Department of Radiation Oncology, Virginia Commonwealth University, Richmond, VA 23298, USA
}

Correspondence should be addressed to Ashraf A. Khalil; ashraf.khalil@virginia.edu

Received 27 August 2013; Accepted 27 October 2013

Academic Editors: H. Hendrikse and P. Lass

Copyright (C) 2013 Ashraf A. Khalil et al. This is an open access article distributed under the Creative Commons Attribution License, which permits unrestricted use, distribution, and reproduction in any medium, provided the original work is properly cited.

Currently, intraperitoneal (IP) injection of D-luciferin is the preferred method of providing substrate for bioluminescence imaging (BLI); however it has a failure rate of 3-10\% due to accidental intestinal injection. The present study evaluates the quality of BLI after subcutaneous (SC) injection of D-luciferin and demonstrates the effectiveness of SC injection in anatomically disparate tumor models. Mice bearing luciferase-expressing tumors underwent BLI after SC or IP injection of D-luciferin. The average time to maximal luminescence was $6 \mathrm{~min}$ (range 5-9 $\mathrm{min}$ ) after SC injection and $8 \mathrm{~min}$ (range 5-8 $\mathrm{min}$ ) after IP injection. Within $7 \mathrm{minutes}$ of injection, SC and IP routes yielded similar luminescence in subcutaneous, intracranial, tongue, and lung xenograft tumor models. In a model of combined subcutaneous and intracranial xenografts, SC injection resulted in proportional luminescence at all sites, confirming that preferential delivery of substrate does not occur. While tumors were occasionally not visualized with IP injection, all tumors were visualized reliably with SC injection. Thus, SC injection of D-luciferin is a convenient and effective alternative to IP injection for BLI in nude mice. It may be a preferable approach, particularly for tumors with weaker signals and/or when greater precision is required.

\section{Introduction}

Bioluminescence is a naturally occurring phenomenon in certain species such as the firefly resulting from oxidation of luciferin to oxyluciferin in the presence of molecular oxygen and adenosine triphosphate. This reaction is catalyzed by the enzyme luciferase and results in the emission of light [1]. Bioluminescence imaging (BLI) is an in vivo optical imaging technique that detects light emitted from firefly luciferaseexpressing cells by special charge coupled device (CCD) camera [2]. This is an extremely valuable experimental tool in cancer biology [3]; noninvasive whole body BLI allows repeated, real-time, and in vivo monitoring of tumors in experimental animal models, regardless of tumor location $[2,4]$. This technique can be used to monitor tumor growth $[5,6]$, cell trafficking [7], protein-protein interactions [8], and response to treatment, including gene therapy and immunotherapy $[9,10]$. Sensitivity of BLI is dependent on various factors including the level of cellular luciferase expression, implantation site of the tumor, and oxygenation and viability of the target tissue [11].

The availability of luciferin at the site of interest is a key element of BLI. D-luciferin can be delivered by intraperitoneal (IP) or intravenous (IV) injection [12, 13], 
but currently IP injection is preferred because of its convenience. D-luciferin is absorbed through the peritoneum and reaches luciferase-expressing cells and tissues via the blood stream. The IP approach carries the risk of injection of D-luciferin into the bowel, which reduces the substrate concentrations at the target tissue resulting in a weaker bioluminescence signal (BLS) $[14,15]$. Additionally, some body organs (e.g., pancreas and spleen) have preferential uptake of D-luciferin after IP injection [16]. These factors may result in inconsistency of luminescent signal and poor test reproducibility; these issues are often not fully recognized [17]. Subcutaneous (SC) injection of D-Luciferin is another route of administration that has been successfully utilized for BLI, for example, in a rat brain tumor model where intensity of BLS was successfully correlated with tumor size $[5,18]$.

Subcutaneous injection is straightforward and carries little or no risk of missed injection, but it has not been extensively evaluated. The present study assesses the utility of SC injection of D-luciferin as an alternative route to IP administration for BLI of xenograft tumors in nude mice. Sequential BLI of nude mice bearing either subcutaneous, intracranial, lung, or tongue xenografts derived from luciferase-expressing cells was performed. The intensity and kinetics of the BLS were evaluated and the results after SC or IP injection were compared. The reproducibility of the BLS on different days was also evaluated and correlated with tumor growth. The study demonstrates that SC injection is an easy and effective route of administration for D-luciferin with a rapid-onset and reproducible BLS regardless of tumor location.

\section{Materials and Methods}

2.1. Cell Lines. U87 human malignant glioma cells and A549 lung adenocarcinoma cells were obtained from ATCC (Manassas, VA). GBM12 cells, generously provided by Dr. C. David James, are derived from a human GBM xenograft model system [19]. All cell lines were transfected with the cDNA encoding firefly luciferase (pGL3 Luciferase, Promega) and selected with G418 to permanently produce U87-Luc, A549-Luc, and GBM12-Luc cell lines. Cells were maintained in DMEM medium (Invitrogen, Grand Island, NY) supplemented with $10 \%$ fetal bovine serum (JRH Biosciences, Lenexa, KS) and 1\% penicillin/streptomycin (Invitrogen). OSC19-Luc head and neck squamous carcinoma cells were kindly provided by Dr. Jeffrey Myers (MD Anderson Cancer Center, Houston, TX) and were maintained in DMEM/F12 medium (Gibco, Grand Island, NY) supplemented with 5\% fetal bovine serum (JRH) and $1 \%$ penicillin/streptomycin (Invitrogen).

2.2. Animals. Xenograft tumors were generated in nude mice and allowed to grow for 7 days before the first BLI was performed. Subsequently, mice underwent BLI at the times specified for each experiment. The mice were handled in accordance with IACUC guidelines; experiments were approved by the institutional Committee for Animal Research. Numbers of mice in each study are indicated in the corresponding figure legends.

Subcutaneous Flank Tumor Model. $1 \times 10^{6}$ U87-Luc cells in $100 \mu \mathrm{L}$ PBS were injected subcutaneously in the dorsal side of the upper hind limb of female nude mice using insulin syringe.

Intracranial Tumor Model. Mice were sedated with $10 \mathrm{mg} / \mathrm{kg}$ ketamine, and a burr hole was made using a Dremel drill approximately $2 \mathrm{~mm}$ lateral and $2 \mathrm{~mm}$ posterior to the intersection of the coronal and sagittal sutures. $1 \times 10^{5}$ GBM12-Luc cells were injected into the brain using a Hamilton syringe at a depth of $3 \mathrm{~mm}$ in a volume of $5 \mu \mathrm{L}$.

Intrathoracic Tumor Model. Mice were sedated with $10 \mathrm{mg} / \mathrm{kg}$ ketamine and $1 \times 10^{6}$ A549-Luc cells in $100 \mu \mathrm{L}$ PBS were injected into the right lung upper lobe through the intercostal muscle using a Hamilton syringe.

Tongue Tumor Model. Mice were sedated with $10 \mathrm{mg} / \mathrm{kg}$ ketamine and $3 \times 10^{4}$ OSC19-Luc cells suspended in $30 \mu \mathrm{L}$ of PBS were injected submucosally into the ventral aspect of the tongue as previously described [20].

2.3. BLI Device and Setting. BLI was performed using the IVIS-200 Imaging System (Xenogen Corporation, Berkeley, CA). Anesthesia was induced with $2 \%$ isoflurane (Abbott Laboratories, Chicago, IL) inhalation in a special air tight transparent anesthesia box for 3-5 min before the mice were moved to the light-tight chamber of the CCD camera in the imaging position. Whereas IP injections of D-luciferin (Xenogen; $150 \mathrm{mg} / \mathrm{kg}$ in $200 \mu \mathrm{L}$ ) were performed immediately before anesthesia, SC injection was performed after anesthetizing and moving the mice in to the imaging chamber. SC injections of D-luciferin (Xenogen; $150 \mathrm{mg} / \mathrm{kg}$ in $200 \mu \mathrm{L}$ ) were performed dorsally in the midline, midway between the head and tail. When IP and SC injection were evaluated in the same group of mice, an interval of at least $3 \mathrm{~h}$ was allowed for complete washout of the substrate. Sequential images were acquired at $1 \mathrm{~min}$ intervals (60 s exposure, no time delay) for at least $30 \mathrm{~min}$. The luminescence camera was set to $60 \mathrm{~s}$ exposure, medium binning, $\mathrm{f} / 1$, blocked excitation filter, and open emission filter. The photographic camera was set to $2 \mathrm{~s}$ exposure, medium binning, and $\mathrm{f} / 8$. Field of view was set to image all mice simultaneously. Identical settings were used to acquire each image and region of interest during the study as previously described [21].

The luminescent area of the xenograft tumor was defined as the region of interest (ROI) and the total signal in the ROI (photon $/ \mathrm{sec} / \mathrm{m}^{2}$ ) was quantified using Living Image software 3D (version 1; Xenogen). The ROI was applied to all images of the same sequence of each tumor. The total signal intensity (photon $/ \mathrm{sec} / \mathrm{m}^{2}$ ) was plotted against time after D-luciferin injection to generate a time intensity curve from which the maximal intensity and time to maximal intensity were determined. 


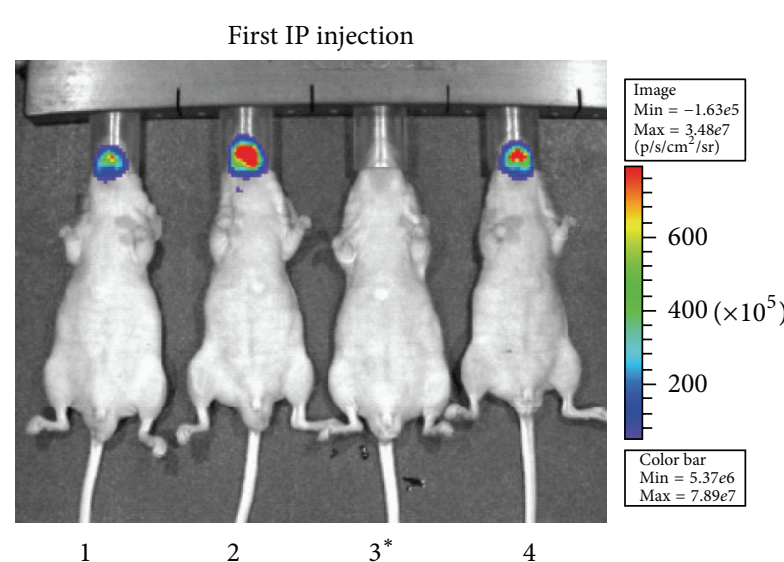

(a)

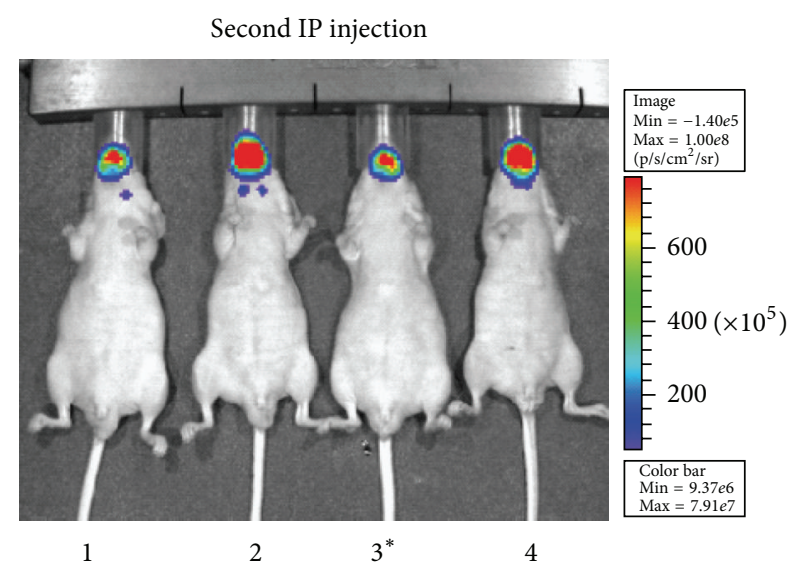

(b)

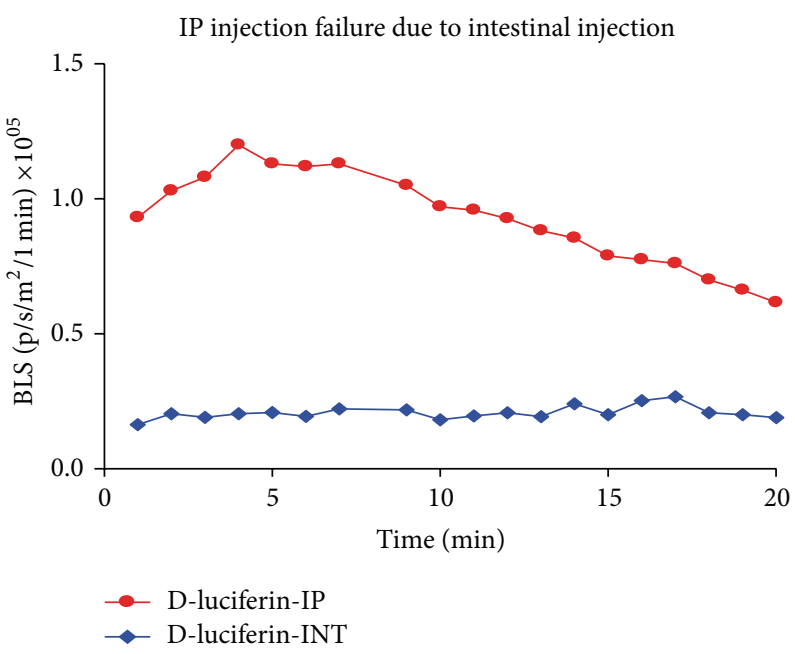

(c)

FIGURE 1: Potential for missed injection when D-luciferin is administered IP. (a) OSC19-Luc tongue xenografts were established in 4 nude mice, and BLI was performed after IP injection of D-luciferin. BLS intensity was evaluated for $1 \mathrm{~min}$ at 7 min after injection. Mouse no. 3 $\left({ }^{*}\right)$ exhibits no BLS due to inadvertent intraintestinal injection. (b) Mouse no. $3\left({ }^{*}\right)$ underwent a second IP administration of D-luciferin and repeat BLI was performed. Mouse no. $3\left({ }^{*}\right)$ exhibited the expected BLS after successful reinjection. (c) A single nude mouse bearing a subcutaneous U87-Luc xenograft underwent BLI after deliberate intraintestinal injection of D-luciferin. BLS was collected continuously at $1 \mathrm{~min}$ intervals as described in Methods. After $3 \mathrm{~h}$, correct IP injection D-luciferin was performed, and BLS was collected as before. The graph shows BLS intensity over time for the intestinal (INT) and IP injections.

2.4. Statistics. The peak signals were compared between SC and IP injections using unpaired 2-tailed $t$-test. Comparisons where $P<0.05$ were considered statistically significant.

\section{Result and Discussion}

3.1. Limitations of IP Injection. The evolution of BLI of human xenografts in immunocompromised mice has been driven by the predominant use of this animal model in cancer biology research. Although most researchers use IP injection to administer D-luciferin for BLI, it may sometimes result in accidental intestinal injection and consequent lack of BLS due to decreased substrate availability at the tumor site. Figure 1 demonstrates the risk of missed injection when using the IP technique. BLI was performed after IP injection of
D-luciferin of 4 nude mice bearing OSC19-Luc orthotopic tongue xenografts. After IP injection of all mice, mouse no. 3 appeared to have no tumor (Figure 1(a)). When all mice were properly reinjected with the substrate, accurate BLI was obtained demonstrating a tongue tumor in mouse no. 3 comparable to the other mice in the group (Figure 1(b)). The absence of an apparent tumor in mouse no. 3 after the initial IP injection was presumably due to inadvertent intraintestinal injection. The rate of IP injection failure has been reported as 3-10\% [14]; if not detected, this error can have a substantial impact on the imaging data and its interpretation. If the error is perceived, performing a second injection will create a lag time that, for applications where a high degree of precision is required, may result in an unacceptable delay. Figure 1(c) shows the kinetics of the BLS in a single mouse bearing a subcutaneous U87-Luc xenograft after 
an intentional intraintestinal injection of D-luciferin compared to the correct IP injection. The tumor produced only minimal BLS intensity after the intraintestinal injection. After a proper IP injection, the BLS signal exhibited the anticipated intensity and showed appropriate time-dependent kinetics.

Another limitation to IP injection technique is the obligatory 3-5 min delay after injection for the induction of inhalational anesthesia before BLI can be performed. The detection of the very early signal could be lost and, if there is a greater delay in the induction of anesthesia, the signal could be weak or undetectable. In the process of quantitative analysis of the intensity of the luminescence, the time of the signal detection after substrate injection represents a crucial factor despite the common use of a predetermined time point to evaluate signal intensity [22]. SC injection is technically simpler than IP and can be performed immediately after the induction of anesthesia with more rapid injection of multiple animals, thus ensuring that the initial luminescence will be detected, that the peak signal will not be missed, and that the peak signal will occur simultaneously in all animals. With SC injection, delivery of the full dose of substrate is ensured, reducing the risk that signal variation is due to injection technique; it can be difficult to determine if a weak signal results from inadequate injection or smaller tumor size, particularly for orthotopic tumors that are not easily palpable.

3.2. Kinetics of BLS Intensity after SC versus IP Injection. The kinetics of BLS intensity were compared in mice bearing subcutaneous U87-Luc xenograft tumors after SC or IP injection of D-luciferin (Figure 2). As described above, there is a $5 \mathrm{~min}$ delay in obtaining images for the animals that underwent IP injection due to performing the injection prior to inducing general anesthesia. The peak BLS after IP injection was observed at $8 \mathrm{~min}$ (range 5-8 $\mathrm{min}$ ); after SC injection the peak luminescence was at $6 \mathrm{~min}$ (range $5-9 \mathrm{~min}$ ). After $30 \mathrm{~min}$, the signals for both injection techniques had fallen by more than $50 \%$. The signals were undetectable after $60 \mathrm{~min}$ (data not shown), indicating complete metabolism of the substrate. With IP injection, luminescent intensity peaked earlier and began to decrease sooner; after SC injection, maximal signal was lower and occurred slightly later, but was sustained much longer at the peak level (approximately $10 \mathrm{~min}$ ).

3.3. Utility of SC Injection at Disparate Anatomic Sites. To evaluate the relative signal quality resulting from SC versus IP injection at different anatomic sites, a series of xenograft models were tested (Figure 3). Multiple mice bearing subcutaneous, intracranial, tongue, and pulmonary xenografts were injected with D-luciferin via the SC or IP route, and the BLS (photon $/ \mathrm{sec} / \mathrm{m}^{2}$ ) was measured $7 \mathrm{~min}$ after injection ( 1 min collection; images in Figures $3(\mathrm{a})-3(\mathrm{~d}))$. For each model, the average BLS intensity was determined for SC and IP injection after 3 distinct imaging sessions. BLS intensity was similar in SC and IP injections in all models, with no significant differences noted (graphs in Figures 3(a)-3(d)). This demonstrates that, compared to IP injection, the SC route of administration results in similar delivery of Dluciferin to the target tissue for all anatomic sites tested.

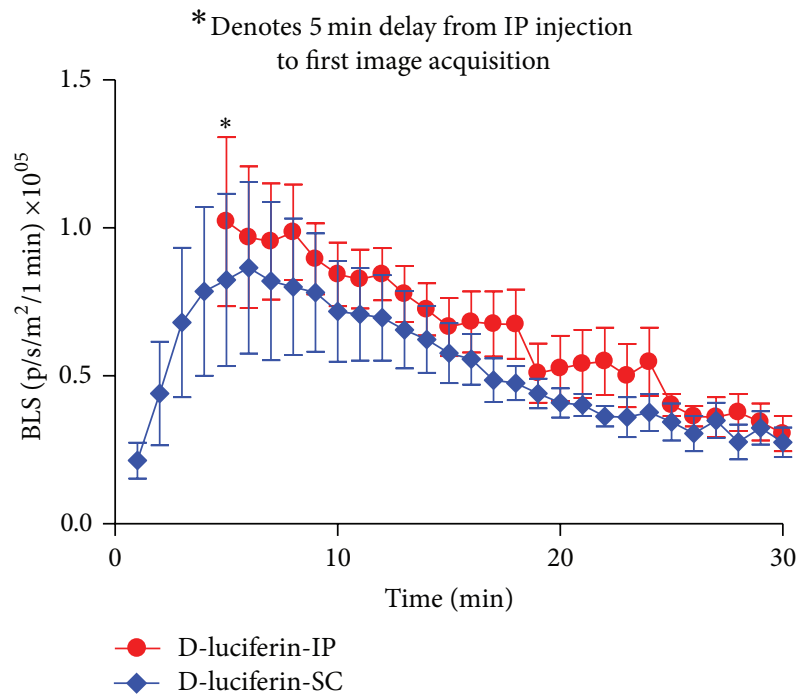

FIGURE 2: Time-intensity curves in various xenograft models. Five nude mice bearing U87-Luc bilateral subcutaneous thigh xenograft tumors underwent BLI after SC or IP injection. BLS was quantified every $\mathrm{min}$ for $30 \mathrm{~min}$. The graph shows average BLS \pm SEM. This study was performed 3 times with similar results.

3.4. SC Injection Does Not Result in Preferential Bioluminescence. A simultaneous subcutaneous and intracranial tumor model was used to determine whether SC injection causes preferential bioluminescence (Figure 4(a)). The BLS emitted from equal size bilateral subcutaneous thigh tumors and an intracranial tumor after SC injection of D-luciferin was plotted against time (Figure 4(b)). The first luminescent signals emitted from the thigh and intracranial tumors were simultaneously detected within the first min after SC injection, and signals from both sites achieved maximal intensity at $10 \mathrm{~min}$. These data confirm that the anatomic location of the tumor does not change the kinetics of luminescence after SC substrate injection. Although D-luciferin has to traverse the blood-brain barrier before it reaches the intracranial tumor [23], simultaneous luminescence from intracranial and thigh xenografts was observed after SC injection. These data are in agreement with Inoue et al. who studied BLI after SC injection of D-luciferin in animals having intraand extraperitoneal cavity tumors [24]. It should be noted that, if there is concern regarding the timing of luminescence in a multilocation tumor model, D-luciferin supplied via intravenous injection could be used to verify the validity of the SC injection approach [25].

3.5. BLI after SC Injection Reflects Tumor Growth. The impact of tumor growth on the time course of luminescence after SC injection was also evaluated. Seven days after implantation, nude mice with U87-Luc subcutaneous thigh xenograft tumors underwent BLI after SC injection with Dluciferin. BLS was acquired every min for $30 \mathrm{~min}$ starting immediately after injection. The procedure was repeated on postimplantation days 7,10 , and 14 . The time to peak signal intensity was consistent after 7, 10, and 14 days of xenograft 

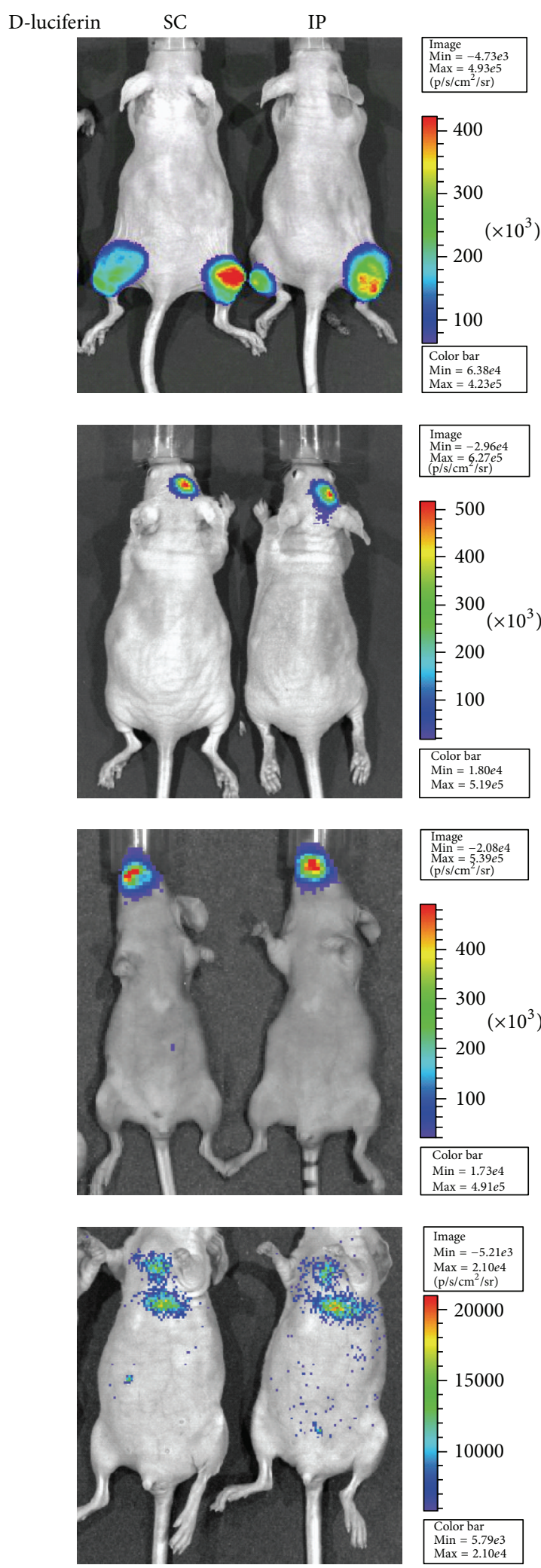

(a)

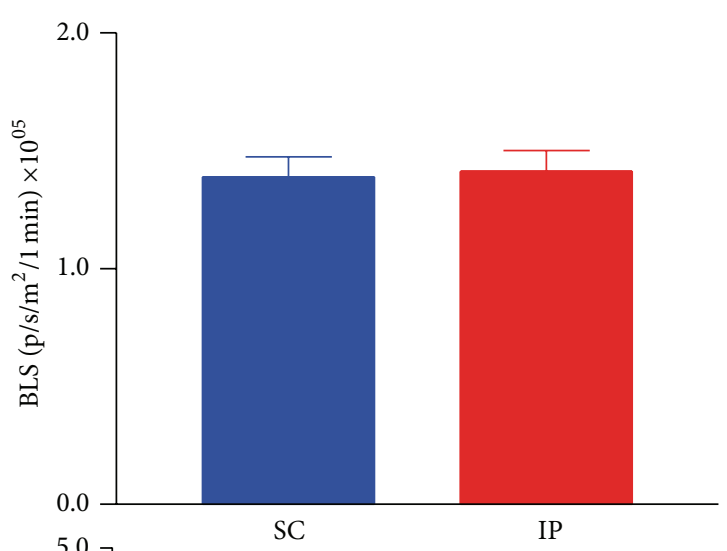

(b)
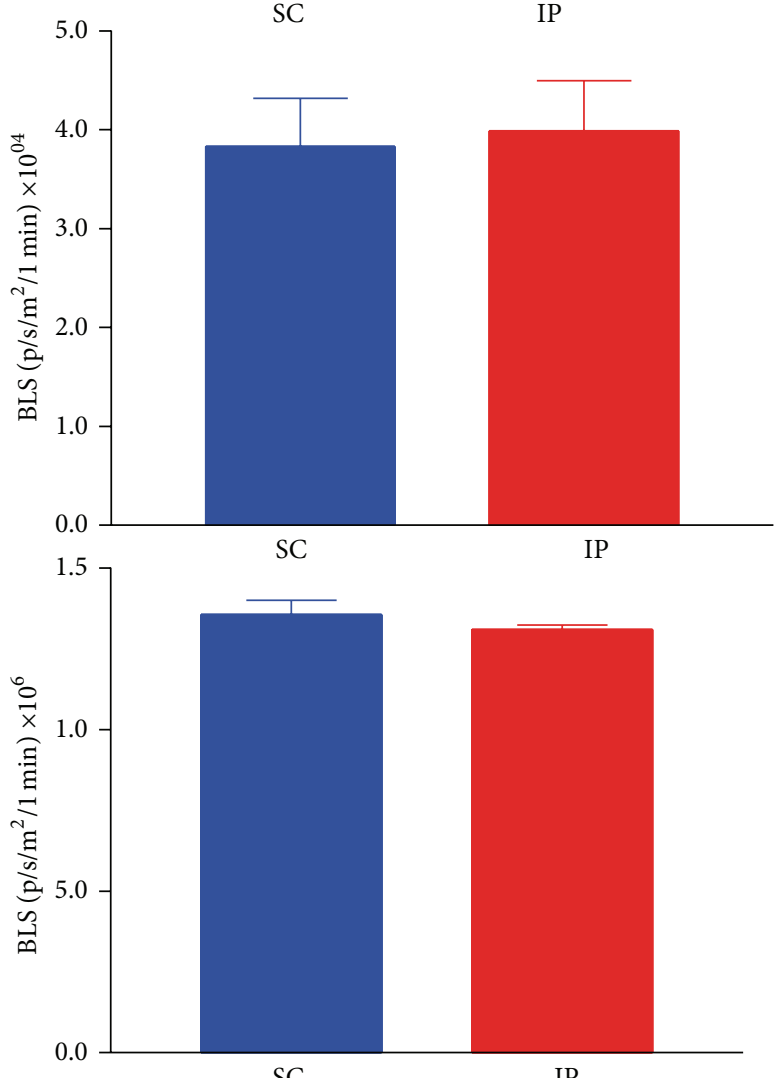

(c)

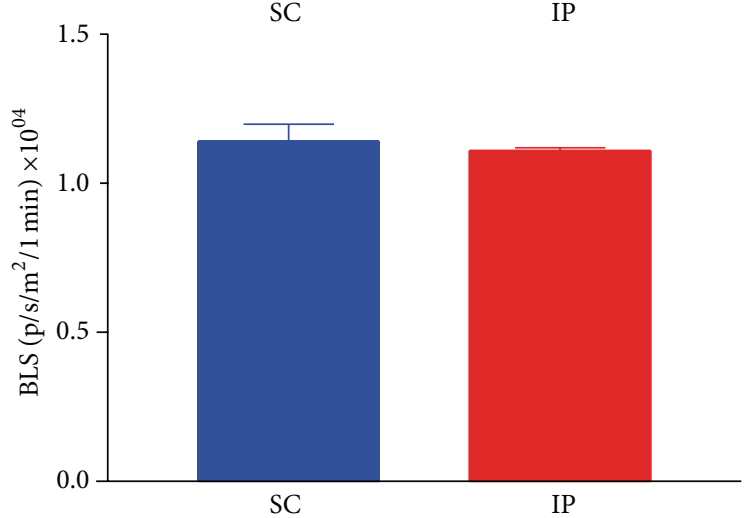

(d)

Figure 3: BLI after SC versus IP injection at different anatomic sites. Nude mice bearing (a) U87-Luc bilateral subcutaneous thigh xenografts (5 mice), (b) GBM12-Luc intracranial brain xenografts (3 mice), (c) OSC19-Luc tongue xenografts (5 mice), or (d) A549-Luc pulmonary xenografts $(2$ mice) were imaged at $7 \mathrm{~min}$ after SC injection and $7 \mathrm{~min}$ after IP injection after an intervening washout period. Shown are representative images after SC (right mouse) or IP (left mouse) injection of D-luciferin. For each anatomic site, the graph shows the average BLS intensity (photon $/ \mathrm{sec} / \mathrm{m}^{2} / 1 \mathrm{~min}$ ) for IP and SC injection \pm SEM after 3 distinct imaging sessions. No statistically significant differences were noted. 

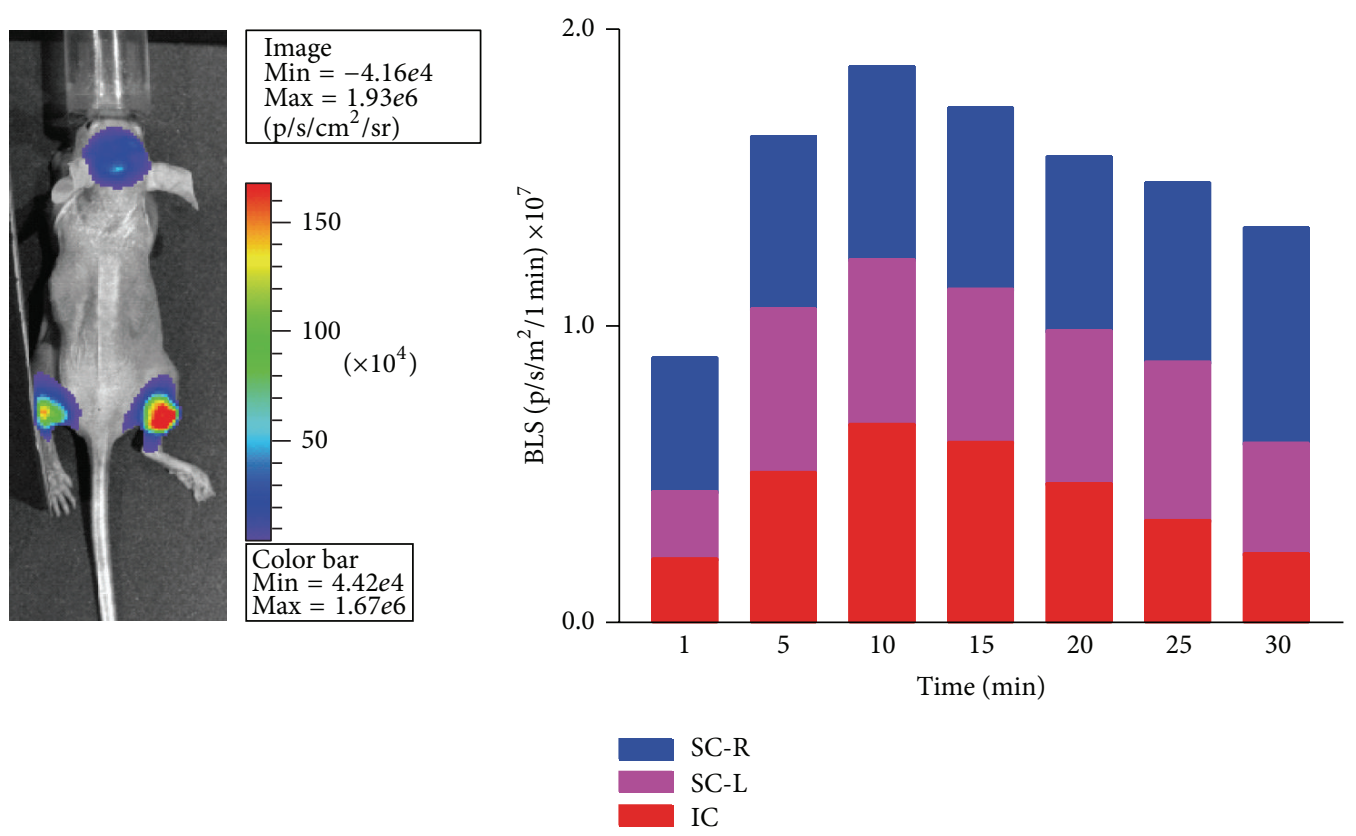

(a)

(b)

FIGURE 4: BLI of multisite xenograft model after SC injection. (a) A single nude mouse bearing bilateral U87-Luc subcutaneous thigh xenograft tumors and, a simultaneous GBM12-Luc intracranial xenograft tumor was imaged 10 min after SC injection of D-luciferin. (b) The BLS of each tumor (1 min collection) was plotted over time.

tumor growth (Figure 5). The peak signal intensity increased as the experiment progressed, confirming the correlation of signal intensity with tumor growth, as has been previously reported $[5,18,21]$. The reproducibility of SC injection appeared to be comparable to or better than IP injection. It should be emphasized that in most BLI studies, to shorten the imaging time, image acquisition is usually obtained at a single, predetermined time point. Using the SC injection approach, the time to the maximal BLS in each sequential imaging pattern did not differ substantially from the mean peak time; thus the assessment of tumor burden by singlepoint imaging at the mean peak time could be expected to yield similar results to previous studies using IP injection $[14,26,27]$.

The data obtained in this study are consistent with previous findings by Inoue et al., but in ours we have additionally tested the application of the SC injection method in a variety of tumor models involving disparate anatomic sites and also in a simultaneous multisite tumor model. The present study also addresses the impact of tumor growth on bioluminescence after SC injection. The kinetics of signal intensity and the relationship between the peak signal and the time after cell implantation demonstrate that the BLS after SC injection is correlated with tumor growth and that the signal kinetics remains unchanged as the tumor enlarges.

\section{Conclusion}

SC injection of D-luciferin is a convenient and effective alternative to IP injection for BLI of xenografts in nude

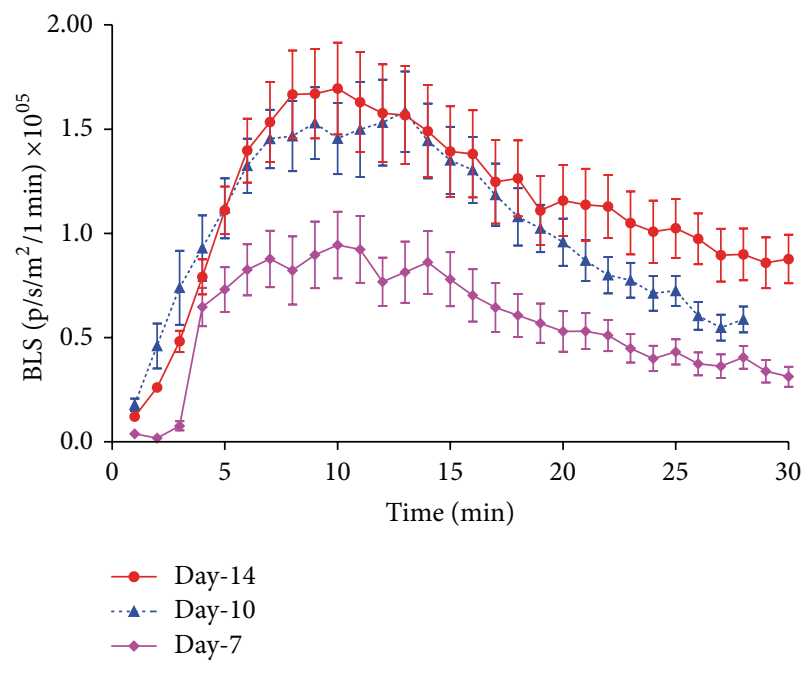

FIGURE 5: BLS intensity after SC injection correlates with tumor growth over time. Five nude mice bearing U87-Luc bilateral subcutaneous thigh xenografts underwent BLI after SC injection of Dluciferin on days 7,10 , and 14 after implantation. BLS was collected every minute for $30 \mathrm{~min}$, and signal intensities were plotted against time on each day the 5 mice were assessed. Error bars indicate SEM.

mice. SC injection is free from risk of injection failure and offers consistent results for BLI of various luciferaseexpressing tissues. SC injection may be a preferable approach, particularly for tumors with weaker signals and/or when 
greater precision is required, or when consistently effective IP injection is more difficult.

\section{References}

[1] E. W. Mc, "Properties of the reaction utilizing adenosinetriphosphate for," The Journal of Biological Chemistry, vol. 191, no. 2, pp. 547-557, 1951.

[2] J.-B. Kim, K. Urban, E. Cochran et al., "Non-invasive detection of a small number of bioluminescent cancer cells in vivo," PLoS ONE, vol. 5, no. 2, Article ID e9364, 2010.

[3] C. E. Badr and B. A. Tannous, "Bioluminescence imaging: progress and applications," Trends in Biotechnology, vol. 29, no. 12, pp. 624-633, 2011.

[4] J. Rousseau, V. Escriou, P. Perrot et al., "Advantages of bioluminescence imaging to follow siRNA or chemotherapeutic treatments in osteosarcoma preclinical models," Cancer Gene Therapy, vol. 17, no. 6, pp. 387-397, 2010.

[5] Y. Zhang, J. P. Bressler, J. Neal et al., "ABCG2/BCRP expression modulates D-luciferin-based bioluminescence imaging," Cancer Research, vol. 67, no. 19, pp. 9389-9397, 2007.

[6] C. H. Contag, D. Jenkins, P. R. Contag, and R. S. Negrin, "Use of reporter genes for optical measurements of neoplastic disease in vivo," Neoplasia, vol. 2, no. 1-2, pp. 41-52, 2000.

[7] A. Nakajima, C. M. Seroogy, M. R. Sandora et al., "Antigenspecific $\mathrm{T}$ cell-mediated gene therapy in collagen-induced arthritis," Journal of Clinical Investigation, vol. 107, no. 10, pp. 1293-1301, 2001.

[8] T. F. Massoud, R. Paulmurugan, A. De, P. Ray, and S. S. Gambhir, "Reporter gene imaging of protein-protein interactions in living subjects," Current Opinion in Biotechnology, vol. 18, no. 1, pp. 3137, 2007.

[9] J. Y. Adams, M. Johnson, M. Sato et al., "Visualization of advanced human prostate cancer lesions in living mice by a targeted gene transfer vector and optical imaging," Nature Medicine, vol. 8, no. 8, pp. 891-896, 2002.

[10] Q. Wang, M. Ornstein, and H. L. Kaufman, "Imaging the immune response to monitor tumor immunotherapy," Expert Review of Vaccines, vol. 8, no. 10, pp. 1427-1437, 2009.

[11] C. P. W. Klerk, R. M. Overmeer, T. M. H. Niers et al., "Validity of bioluminescence measurements for noninvasive in vivo imaging of tumor load in small animals," BioTechniques, vol. 43, no. 1, pp. 7-30, 2007.

[12] M. Edinger, P. Hoffmann, C. H. Contag, and R. S. Negrin, "Evaluation of effector cell fate and function by in vivo bioluminescence imaging," Methods, vol. 31, no. 2, pp. 172-179, 2003.

[13] R. Tuli, A. Surmak, J. Reyes et al., "Development of a novel preclinical pancreatic cancer research model: bioluminescence image-guided focal irradiation and tumor monitoring of orthotopic xenografts," Translational Oncology, vol. 5, no. 2, pp. 7784, 2012.

[14] Z. Paroo, R. A. Bollinger, D. A. Braasch et al., "Validating bioluminescence imaging as a high-throughput, Quantitative modality for assessing tumor burden," Molecular Imaging, vol. 3, no. 2, pp. 117-124, 2004.

[15] Y. Inoue, S. Kiryu, K. Izawa, M. Watanabe, A. Tojo, and K. Ohtomo, "Comparison of subcutaneous and intraperitoneal injection of d-luciferin for in vivo bioluminescence imaging," European Journal of Nuclear Medicine and Molecular Imaging, vol. 36, no. 5, pp. 771-779, 2009.
[16] K.-H. Lee, S. S. Byun, J.-Y. Paik et al., "Cell uptake and tissue distribution of radioiodine labelled D-luciferin: implications for luciferase based gene imaging," Nuclear Medicine Communications, vol. 24, no. 9, pp. 1003-1009, 2003.

[17] M. Keyaerts, J. Verschueren, T. J. Bos et al., "Dynamic bioluminescence imaging for quantitative tumour burden assessment using IV or IP administration of d-luciferin: effect on intensity, time kinetics and repeatability of photon emission," European Journal of Nuclear Medicine and Molecular Imaging, vol. 35, no. 5, pp. 999-1007, 2008.

[18] M. J. Bryant, T. L. Chuah, J. Luff, M. F. Lavin, and D. G. Walker, "A novel rat model for glioblastoma multiforme using a bioluminescent F98 cell line," Journal of Clinical Neuroscience, vol. 15, no. 5, pp. 545-551, 2008.

[19] C. Giannini, J. N. Sarkaria, A. Saito et al., "Patient tumor EGFR and PDGFRA gene amplifications retained in an invasive intracranial xenograft model of glioblastoma multiforme," Neuro-Oncology, vol. 7, no. 2, pp. 164-176, 2005.

[20] J. N. Myers, F. C. Holsinger, S. A. Jasser, B. N. Bekele, and I. J. Fidler, "An orthotopic nude mouse model of oral tongue squamous cell carcinoma," Clinical Cancer Research, vol. 8, no. 1, pp. 293-298, 2002.

[21] A. A. Khalil, M. J. Jameson, W. C. Broaddus et al., "The influence of hypoxia and $\mathrm{pH}$ on bioluminescence imaging of luciferasetransfected tumor cells and xenografts," International Journal of Molecular Imaging, vol. 2013, Article ID 287697, 9 pages, 2013.

[22] Y. Inoue, S. Kiryu, M. Watanabe, A. Tojo, and K. Ohtomo, "Timing of imaging after D-luciferin injection affects the longitudinal assessment of tumor growth using in vivo bioluminescence imaging," International Journal of Biomedical Imaging, vol. 2010, Article ID 471408, 6 pages, 2010.

[23] A. Sun, L. Hou, T. Prugpichailers et al., "Firefly luciferase-based dynamic bioluminescence imaging: a noninvasive technique to assess tumor angiogenesis," Neurosurgery, vol. 66, no. 4, pp. 751757, 2010.

[24] Y. Inoue, A. Tojo, R. Sekine et al., "In vitro validation of bioluminescent monitoring of disease progression and therapeutic response in leukaemia model animals," European Journal of Nuclear Medicine and Molecular Imaging, vol. 33, no. 5, pp. 557$565,2006$.

[25] W. Wang and W. S. El-Deiry, "Bioluminescent molecular imaging of endogenous and exogenous p53-mediated transcription in vitro and in vivo using an HCT116 human colon carcinoma xenograft model," Cancer Biology and Therapy, vol. 2, no. 2, pp. 196-202, 2003.

[26] S. Baba, S. Y. Cho, Z. Ye, L. Cheng, J. M. Engles, and R. L. Wahl, "How reproducible is bioluminescent imaging of tumor cell growth? Single time point versus the dynamic measurement approach," Molecular Imaging, vol. 6, no. 5, pp. 315-322, 2007.

[27] M. G. Abdelwahab, T. Sankar, M. C. Preul, and A. C. Scheck, "Intracranial implantation with subsequent 3D in vivo bioluminescent imaging of murine gliomas," Journal of Visualized Experiments, no. 57, article e3403, 2011. 

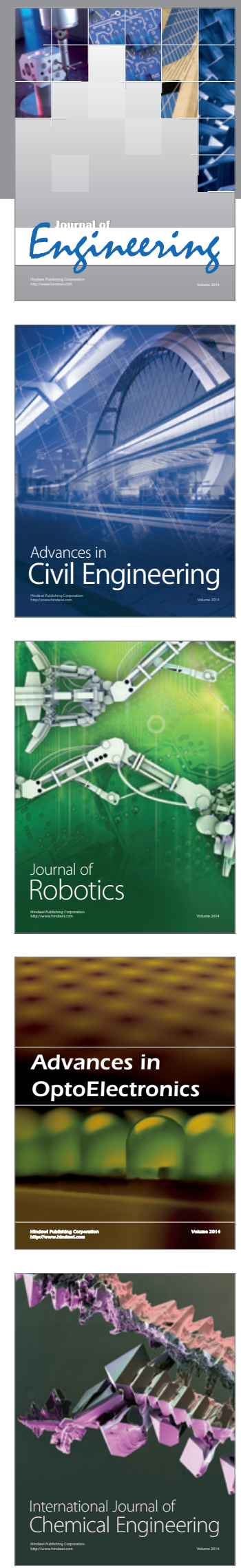

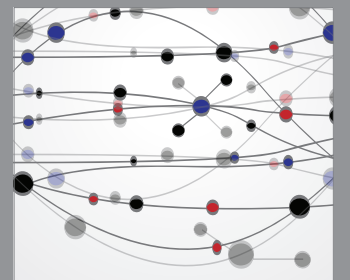

The Scientific World Journal
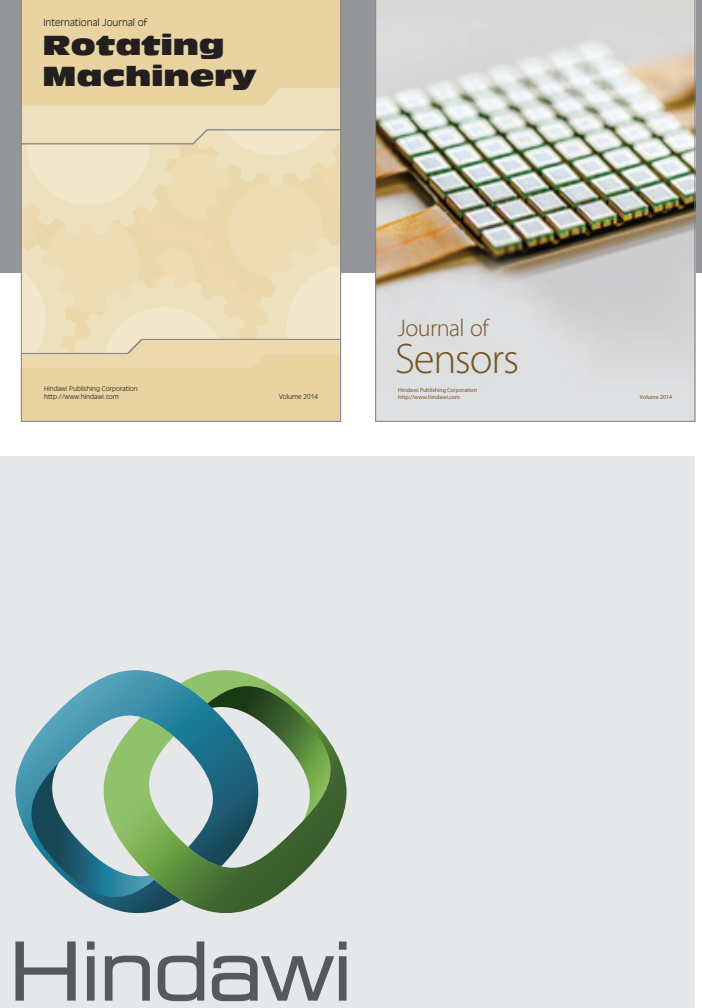

Submit your manuscripts at http://www.hindawi.com
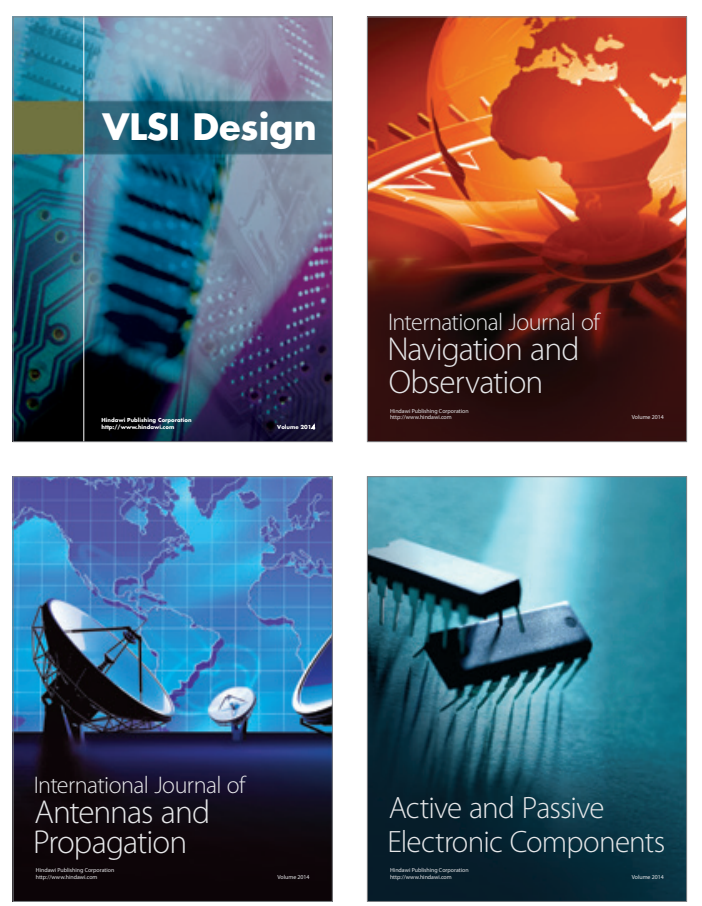
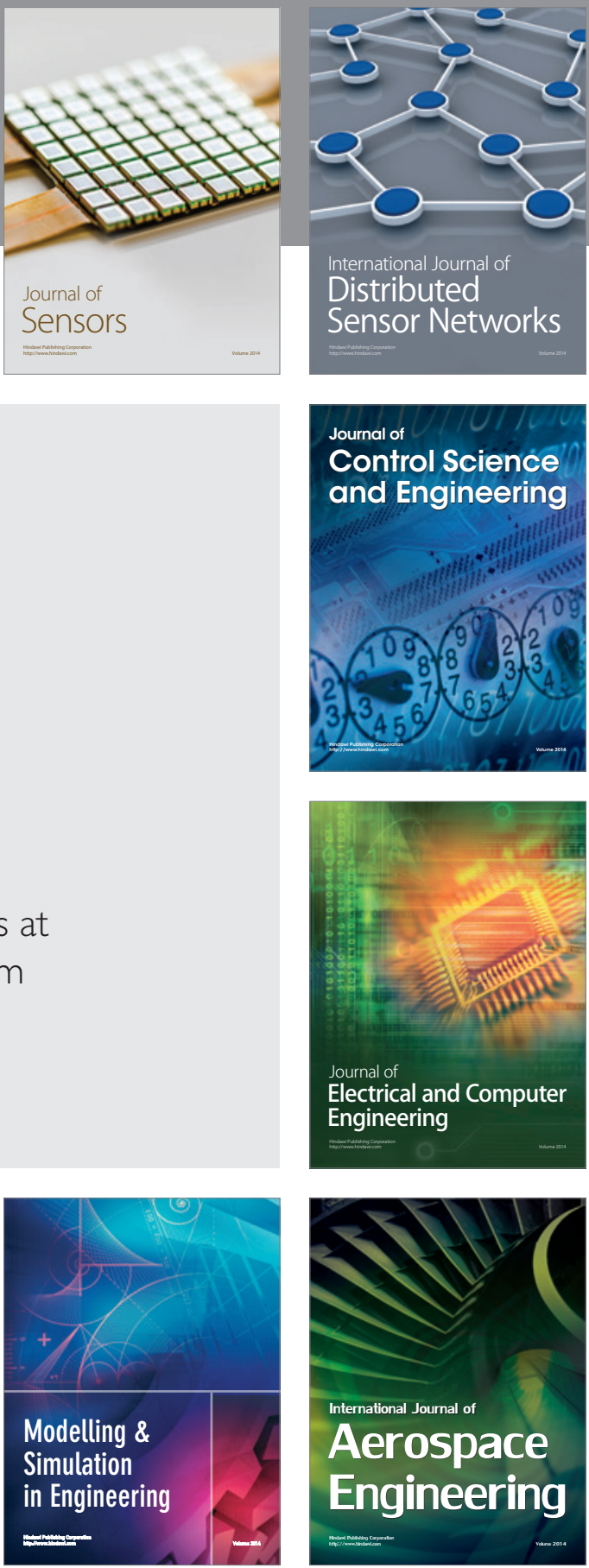

Journal of

Control Science

and Engineering
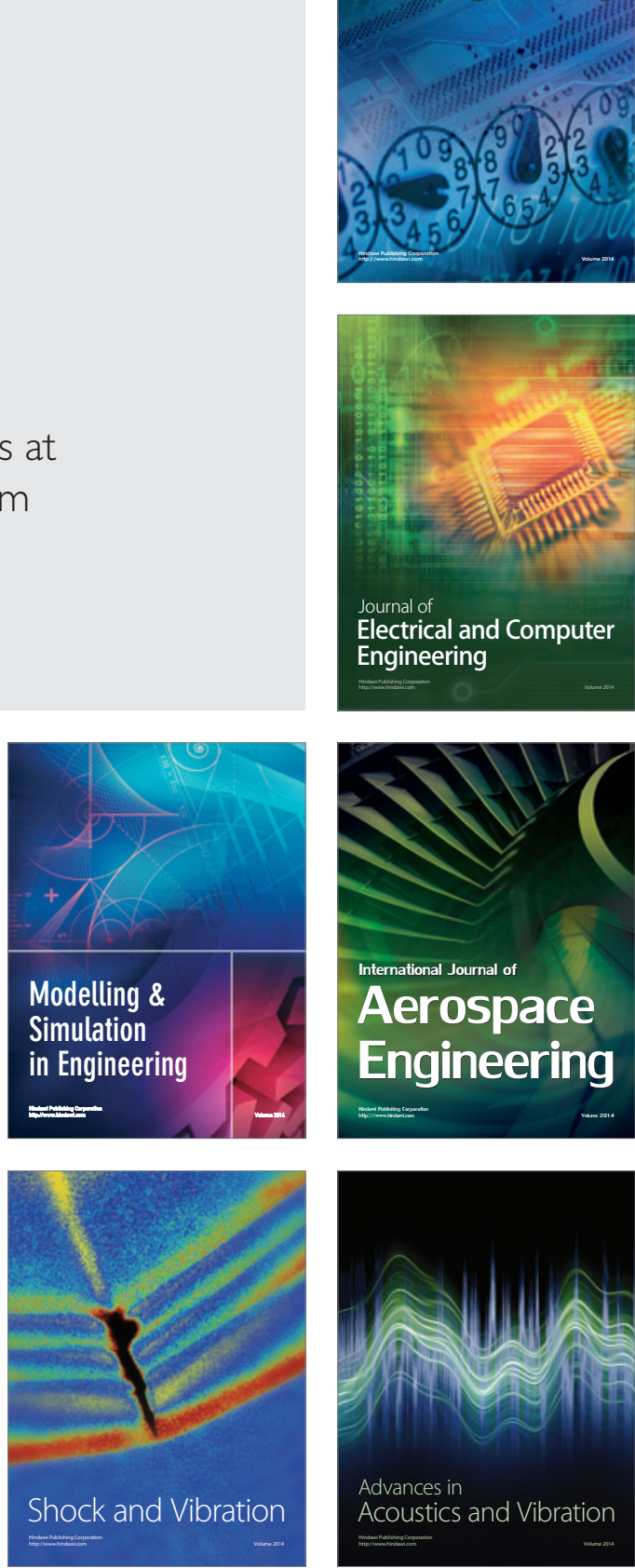